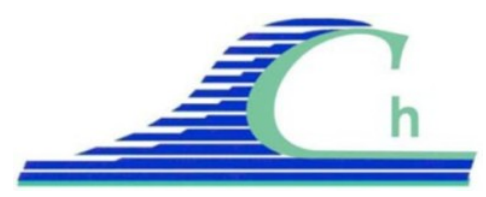

XII ${ }^{\text {èmes }}$ Journées Nationales Génie Côtier - Génie Civil

Cherbourg, 12-14 juin 2012

DOI:10.5150/jngcgc.2012.073-C @ Editions Paralia CFL

disponible en ligne - http://www.paralia.fr - available online

\title{
Le diagnostic d'ouvrages portuaires anciens : un outil d'aide à la gestion
}

\section{Bertrand COLLIN ${ }^{1}$, Frédéric CAPPELAERE ${ }^{2}$, Vincent JOUVE $^{3}$}

1. SITES SAS 2 bis, avenue du Centre 92500 Rueil Malmaison, France. bertrand.collin@sites.fr

2. SITES SAS, entité Nord Pas de Calais, France.

3. SITES SAS, entité Rhône Alpes, France.

\section{Résumé :}

Les gestionnaires d'ouvrages portuaires anciens, qui constituent la majorité du parc actuellement en service, doivent faire face à plusieurs problèmes : vieillissement des matériaux et des structures, risques accrus d'évènements climatiques exceptionnels, maintien -voire augmentation- du niveau de service, adaptation des structures à de nouveaux équipements portuaires.

L'évaluation de l'état et du comportement des structures portuaires anciennes au regard de ces contraintes est donc un élément essentiel d'aide à la décision quant aux choix stratégiques, techniques et économiques à opérer pour assurer la continuité de l'exploitation.

Cette évaluation passe par la réalisation d'un diagnostic qui doit fournir à l'exploitant les éléments objectifs lui permettant d'opérer ces choix.

La présentation de deux études représentatives réalisées sur deux ouvrages anciens -un quai céréalier à La Rochelle et un appontement pétrolier à la Guadeloupe- permet d'illustrer la démarche du diagnostic à partir de deux exemples concrets.

Dans chacun de ces deux exemples, la problématique du gestionnaire est exposée, et les moyens et méthodes utilisés pour y répondre sont présentés : étude documentaire, inspections aériennes et subaquatiques, relevés géométriques, instrumentation à court et moyen terme, évaluation et essais non destructifs sur les matériaux constitutifs, modélisation numérique.

Les conclusions issues de ces études de diagnostics sont ensuite commentées, en s'attachant à relier ces cas particuliers à des problématiques plus larges de maintien en exploitation de structures vieillissantes et parfois endommagées, et en proposant des méthodes de suivi globales qui intègrent les développements les plus récents dans le domaine de l'évaluation des structures.

\section{Mots-clés :}

Ouvrages portuaires anciens - Béton, acier - Vieillissement - Contraintes d'exploitation Diagnostic - Essais non destructifs - Instrumentation - Modélisation - Evaluation 


\section{Introduction}

Avec sa riche histoire maritime, la France possède un grand patrimoine d'ouvrages portuaires. Ces ouvrages, distribués sur l'ensemble du littoral métropolitain et ultramarin, présentent une importante diversité. Ils sont exposés à une grande variété de conditions environnementales qui vont du polaire au tropical et qui incluent des risques sismiques et cycloniques.

Les gestionnaires d'installations portuaires se doivent d'assurer une exploitation optimale en minimisant les coûts de maintenance et les arrêts d'exploitation. De plus, les installations doivent pouvoir prendre en charge de nouveaux équipements, traiter des tonnages de plus en plus importants et répondre aux exigences d'un référentiel normatif en évolution.

Dans ces conditions, il incombe aux gestionnaires de connaître parfaitement l'état de leur patrimoine afin de rationaliser les actions de maintenance et d'être à même de répondre rapidement aux demandes de modifications de niveau de service et de prolongation de durée de vie.

En raison de l'âge des infrastructures et des changements des politiques de gestion des grands ports maritimes, il est rare de pouvoir disposer d'un historique continu et complet sur un parc de structures portuaires. Le plus souvent, pour répondre à une demande de changement de destination, d'augmentation de niveau de service ou de prolongation de durée de vie, le gestionnaire doit "repartir de zéro" ; il lui faut connaître l'état réel de la structure, évaluer ses performances et sa conformité vis-à-vis des normes en vigueur pour étudier ensuite la faisabilité du projet.

Pour illustrer cette problématique, nous avons choisi de présenter deux exemples : un quai céréalier en Charente Maritime et un appontement pétrolier en Guadeloupe. Dans le premier cas, le gestionnaire a été amené à engager des études de diagnostic pour examiner dans quelle mesure son ouvrage pouvait accepter une modification de niveau de service et pour déterminer les actions nécessaires à garantir en toute sécurité ces nouvelles exigences. Dans le deuxième cas, il s'agissait vérifier l'état, les performances et la conformité d'un ouvrage ancien en vue de la prolongation de sa durée de vie, puis d'établir les préconisations adaptées à cet objectif.

\section{Présentation des ouvrages et des projets}

\subsection{Le quai Modéré Lombard du Grand Port Maritime de La Rochelle}

Réalisée entre 1987 et 1988, l'extension du quai Modéré Lombard devait, en permettant l'installation d'un portique de 520 tonnes et la reprise d'efforts d'accostage et d'amarrage de 100 tonnes, augmenter les performances de ce quai ancien et hétérogène. Cette extension, d'une longueur totale de 288 mètres et d'une largeur de 14,50 mètres, comporte deux files de pieux métalliques, chaque file étant couronnée par une poutre en béton armé constituée d'éléments préfabriqués clavés par bétonnage sur les pieux. Les 


\section{XII ${ }^{\text {èmes }}$ Journées Nationales Génie Côtier - Génie Civil \\ Cherbourg, 12-14 juin 2012}

deux poutres, équipées de rails, forment une voie de grue d'espacement 11,70 mètres. La couverture du quai est assurée par des caissons préfabriqués en "U" dont les nervures font office d'entretoises. L'extension du quai est reliée à une digue de protection par cinq passerelles transversales en béton armé, chaque passerelle, d'une longueur de 30,50 mètres et d'une largeur de 7,6 mètres, comportant deux travées continues reposant sur une culée côté digue et sur une pile en mer.

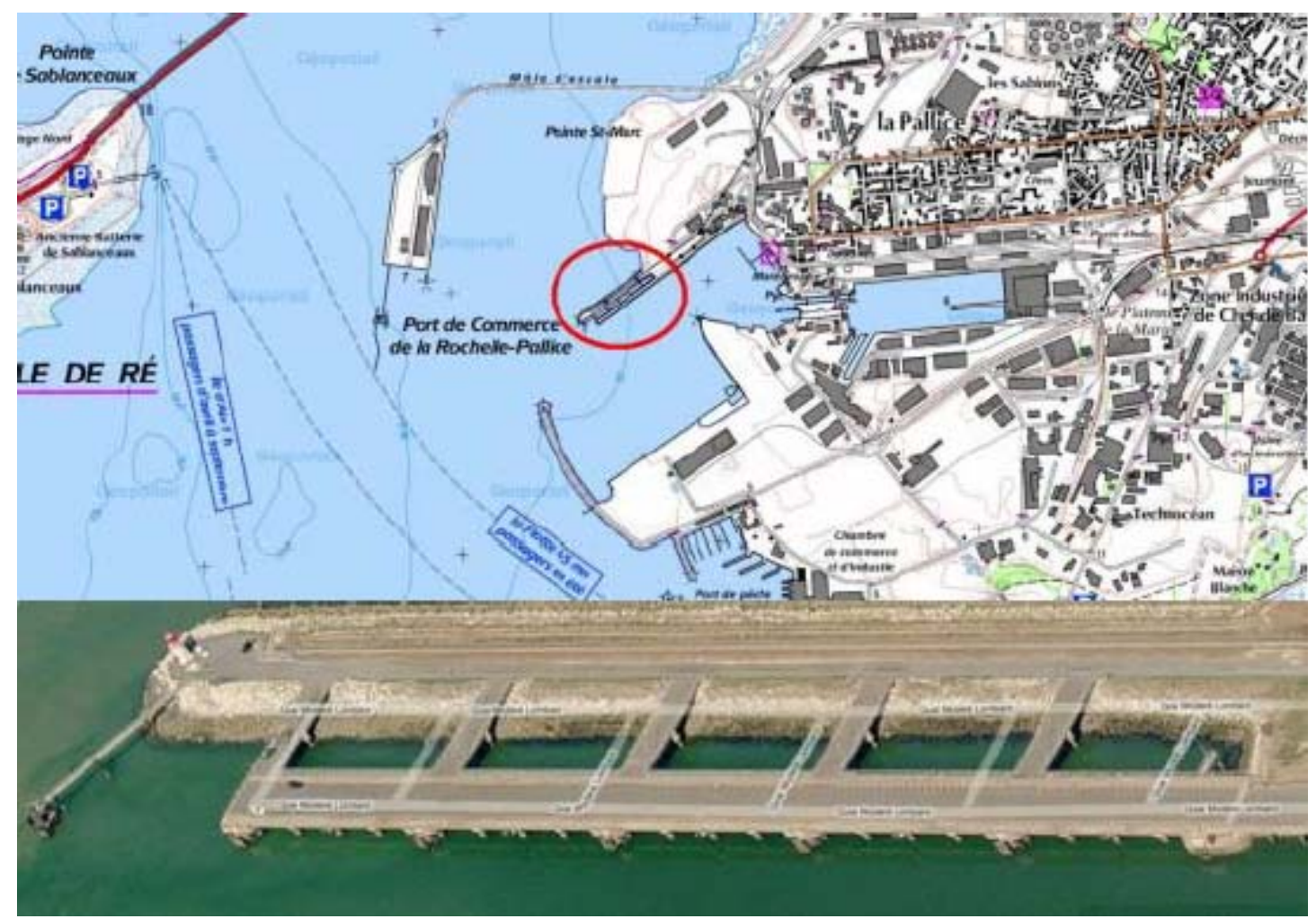

Figure 1. Implantation et vue générale du quai Modéré Lombard.

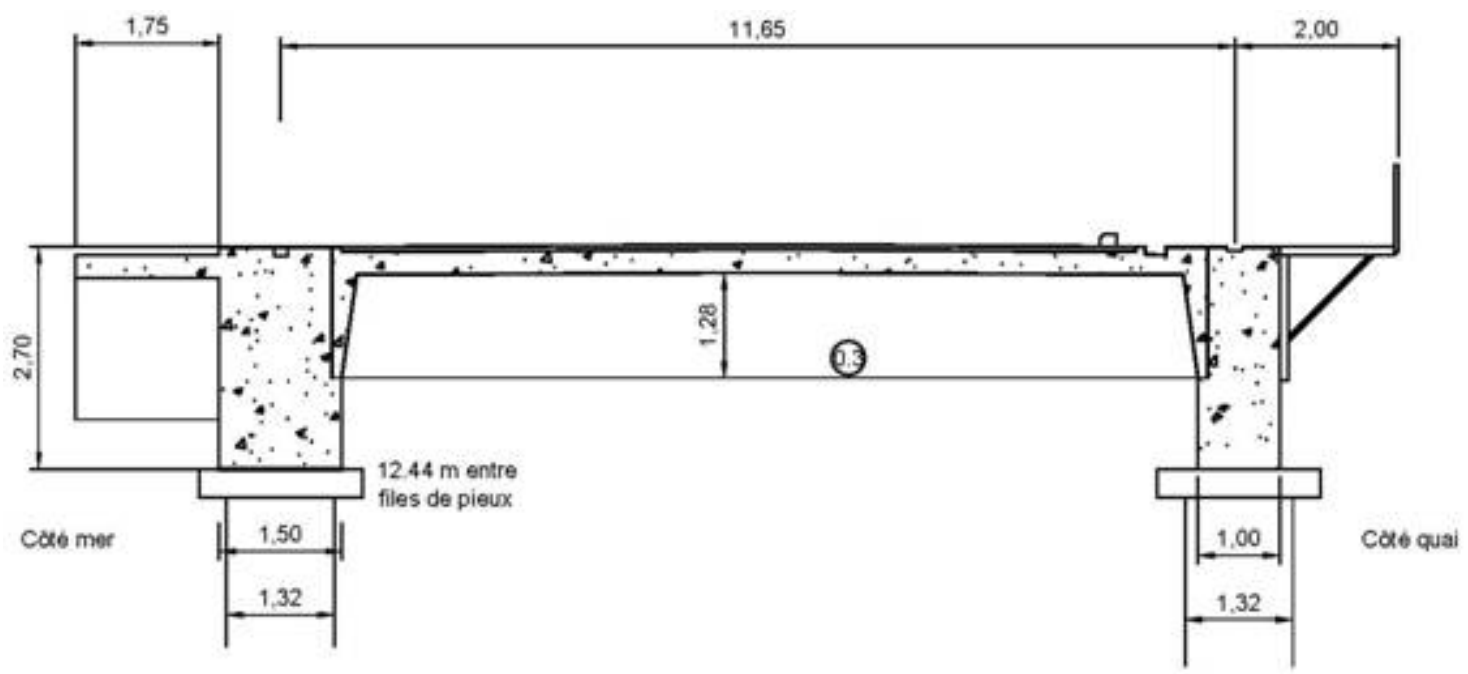

Figure 2. Coupe en travers type du quai. 


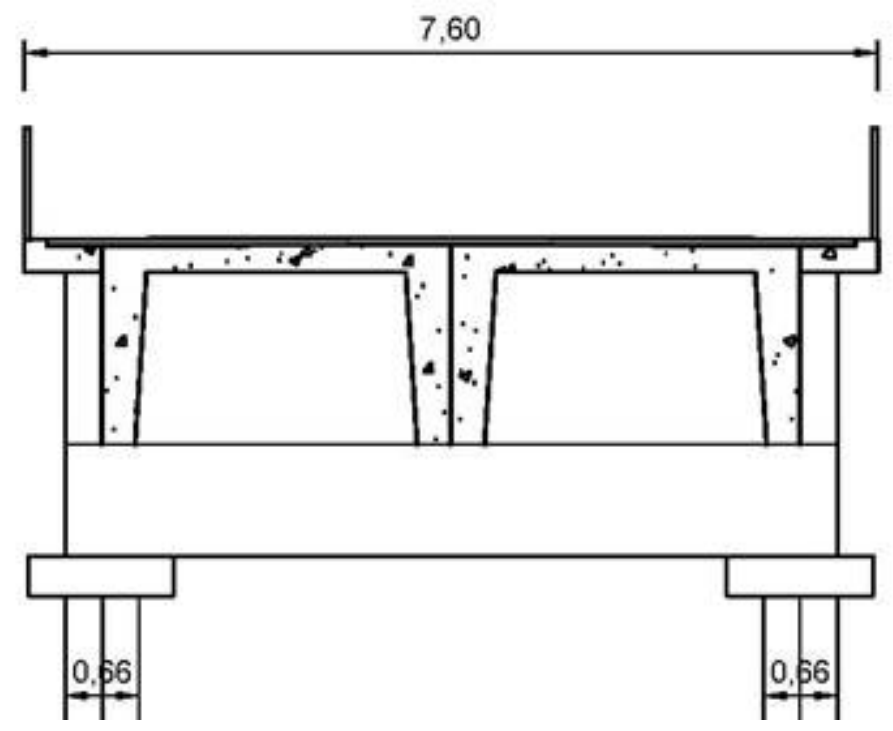

Figure 3. Coupe en travers type des passerelles.

Pour valider la faisabilité du remplacement du portique actuel d’une capacité de 92 tonnes par des ouvrages plus performants, le Grand Port Maritime de La Rochelle a fait procéder à plusieurs vérifications : en 2006, pour une grue sur rails d'une capacité de 480 tonnes et en 2010, pour un portique d’une capacité de 380 tonnes. Ces calculs ont montré que le dimensionnement originel, établi avec les règles de calcul du B.A.E.L. 83, ne répondait pas aux exigences règlementaires actuelles du B.A.E.L. 91 : pas de prise en compte du retrait du béton, traitement distinct de la flexion longitudinale et de la traction, pas de prise en compte des effets de torsion lors des accostages, dépassement systématique de la capacité réglementaire au droit des zones d'accostage. En conclusion, il était proposé d'autoriser le remplacement du portique de 92 tonnes par un portique de 380 tonnes sous réserve de la vérification du "bon état" du quai (pas de fissuration mécanique importante ou de zones d'armatures mises à nu et corrodées) et de limitations d'exploitation (stationnement du portique hors ouvrage lors de l'accostage des navires de fort tonnage, positionnement du portique entre les axes des passerelles lors de l'accostage des navires de plus faible tonnage).

L’étude de diagnostic présentée ici a été engagée par le Grand Port Maritime de La Rochelle pour déterminer à quelles conditions ces réserves pouvaient être levées.

\subsection{L’appontement pétrolier de la pointe Jarry à la Guadeloupe}

L'exploitation du quai $n^{\circ} 9$ de la centrale thermique de Jarry Nord fait partie du projet de modernisation de ce centre de production d'électricité. Cet appontement, construit en 1983, comporte trois ensembles de structures à poutres préfabriquées en béton armé entretoisées sur appuis et reposant sur des pieux métalliques : une passerelle d'accès à onze travées d'une longueur de 165 mètres pour une largeur de 5,50 mètres, une zone de retournement de 17,75 mètres pour une largeur de 11,40 mètres et une plate-forme de 


\section{XII ${ }^{\text {èmes }}$ Journées Nationales Génie Côtier - Génie Civil \\ Cherbourg, 12-14 juin 2012}

déchargement d'une longueur de 18,50 mètres pour une largeur de 7,15 mètres. Un Duc d'Albe en béton armé d'une longueur de 7 mètres pour une largeur de 6,06 mètres complète l'ensemble.

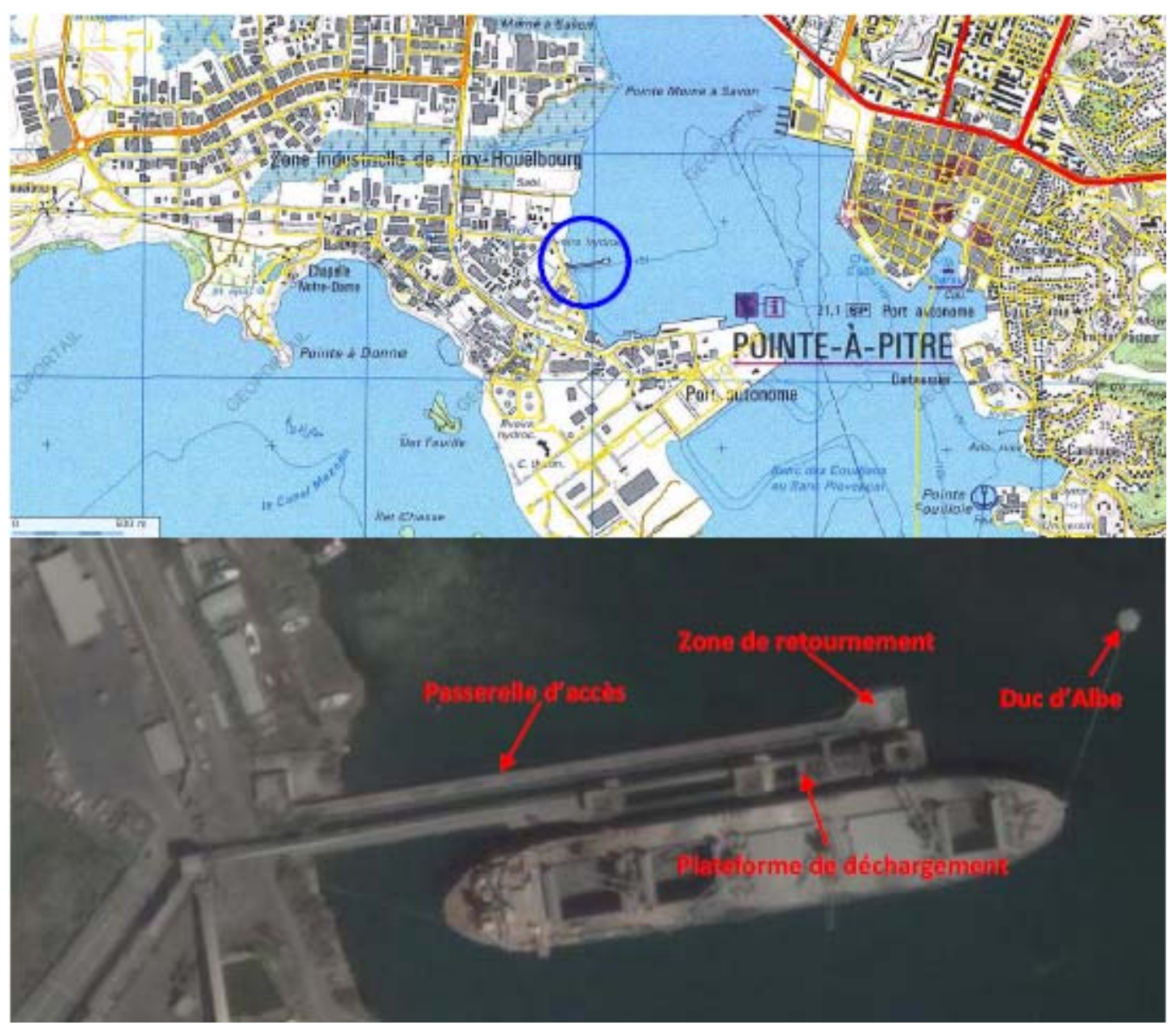

Figure 4. Implantation et vue générale du quai $n^{\circ} 9$.

Compte tenu de l'âge de cet ouvrage et des désordres constatés par l'exploitant, EDF a souhaité engager des études pour savoir à quelles conditions l'exploitation du quai pouvait être durablement poursuivie.

Ces études comprenaient dans un premier temps une expertise de l'état de l'ouvrage, la vérification de ses performances vis-à-vis des charges d'exploitation et des règles actuelles de calcul cyclonique et sismique.

Dans un deuxième temps, suite à ce diagnostic, des préconisations devaient être établies pour restaurer le niveau de service de l'appontement, avec un objectif de durabilité de trente ans. 


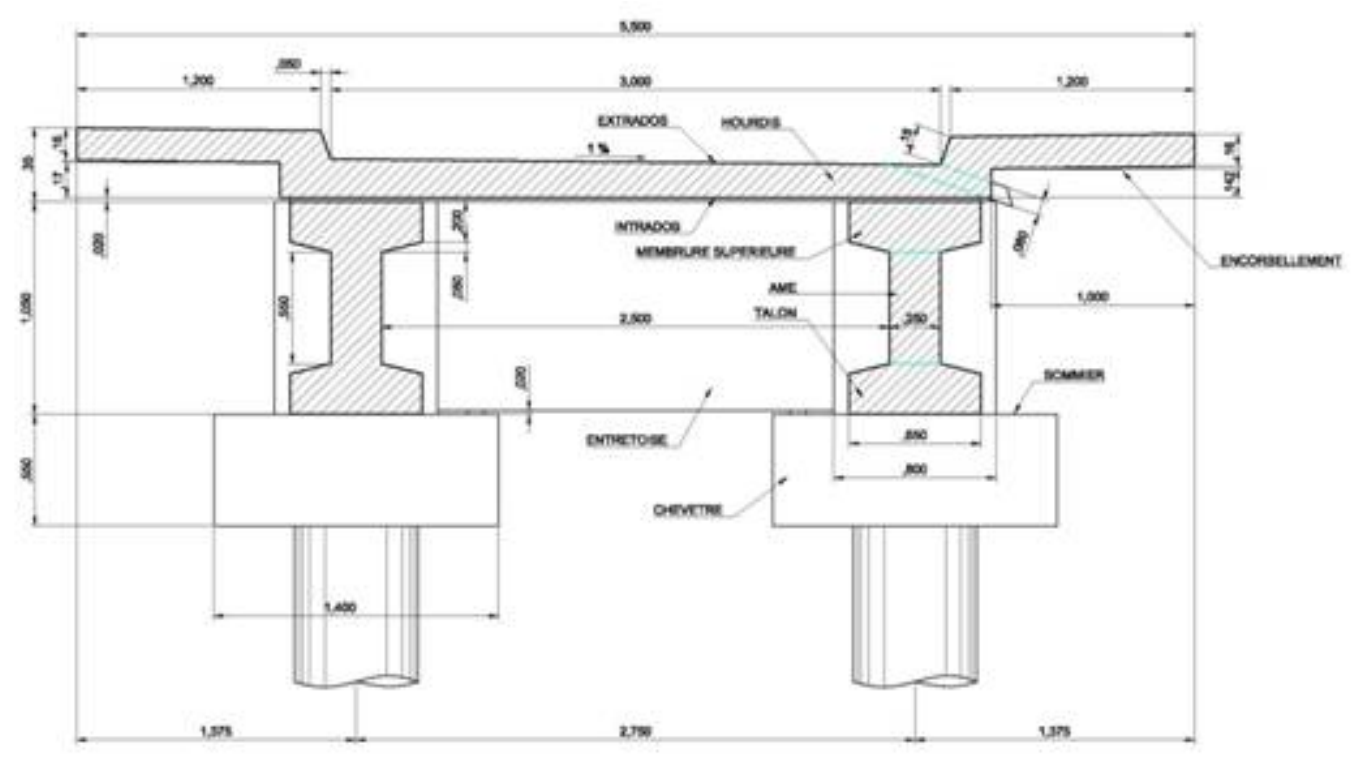

Figure 5. Coupe type sur passerelle d'accès.

\section{Diagnostic et préconisations}

\subsection{Problématique générale du diagnostic}

Le but du diagnostic est de fournir des éléments techniques objectifs pour évaluer l'état d'une structure, proposer les préconisations nécessaires à la remise à niveau et examiner la faisabilité des projets de prolongation de durée de vie, d'aménagement ou de changement de destination.

Le diagnostic d'une structure ancienne doit intégrer et associer des informations relatives aux matériaux et à la structure. Le diagnostic "matériaux" est établi à partir de mesures réalisées in situ et sur prélèvements. Il est destiné à évaluer les caractéristiques des matériaux constitutifs - essentiellement béton et acier - sur la base d'investigations menées sur des zones considérées comme représentatives de l'ensemble de la structure. Le diagnostic "structure" est élaboré en combinant l'analyse de relevés de défauts, de mesures géométriques et de modélisations numériques. Il aboutit à un avis sur les performances et le fonctionnement réels de la structure et permet d'identifier des anomalies de fonctionnement.

D’un point de vue pratique, établir le diagnostic d'un ouvrage portuaire ancien présente un certain nombre de difficultés : documents entrants souvent incomplets, difficultés d'accès, contraintes d'exploitation, choix de l'échantillonnage des zones à investiguer, prise en compte des évolutions des règlements de calcul, référentiels techniques parfois flous en ce qui concerne le diagnostic "matériaux" et contraintes budgétaires.

Le programme de diagnostic doit être élaboré en tenant compte de ces différents éléments, parfois incompatibles, tout en gardant pour objectif l'obtention de résultats exploitables et fiables. 


\section{XII ${ }^{\text {èmes }}$ Journées Nationales Génie Côtier - Génie Civil \\ Cherbourg, 12-14 juin 2012}

Etablir directement des préconisations de remise en état ou de renforcement structurels à partir d'un diagnostic n'est pas toujours chose aisée car il peut subsister, après la réalisation du programme de diagnostic, des inconnues qui, au mieux, peuvent être levées par des investigations complémentaires (essais de chargement, mesures de paramètres spécifiques, ...) et, au pire, sont découvertes en cours de chantier. L'existence de ces inconnues résulte souvent de l'absence de données sur la vie de l'ouvrage ou d'un programme de diagnostic trop restreint en termes de durée et d'étendue.

Les programmes d'investigations mis en œuvre pour le quai Modéré Lombard et l'appontement de Jarry Nord, présentés dans les deux paragraphes suivants, illustrent cette problématique.

\subsection{Extension du quai Modéré Lombard}

La programme de diagnostic a été établi à partir de l'analyse des données d'entrée disponibles -phasage de construction, plans d'exécution, études successives de faisabilité de remplacement du portique-, d'échanges avec le gestionnaire et d'une visite préliminaire du site. Ce programme comportait :

- une inspection détaillée complète des parties émergées, destinée à relever les défauts existants sur l'ensemble des éléments de structure et des équipements; les informations fournies par cette inspection devaient permettre de vérifier si les réserves quant au "bon état" pouvaient être levées et devaient guider le choix des zones à investiguer au titre du diagnostic "matériau" ;

- la vérification d’un certain nombre de données géométriques, sur des zones identifiées comme sensibles du point de vue du fonctionnement ;

- la vérification de l'état de vieillissement du béton armé, décidée en raison des conditions environnementales et des défauts observés lors de la visite préliminaire du site.

Tableau 1. Vérification des données géométriques du quai Lombard.

\begin{tabular}{lcc}
\hline Objet du contrôle & Zones investiguées & Techniques utilisées \\
\hline $\begin{array}{l}\text { Cotes principales } \\
\text { Enrobage des armatures }\end{array}$ & Poutres principales et caissons & Mesure directe sur site \\
\hline $\begin{array}{l}\text { Ferraillage longitudinal des poutres } \\
\text { principales }\end{array}$ & Poutre côté mer et poutre côté quai & RADAR et sondages \\
Ferraillage transversal des poutres & Sur appui et à mi portée \\
principales & RADAR et sondages \\
Résistance en compression du béton & Poutres principales & Scléromètre \\
\hline
\end{tabular}


Tableau 2. Vérification de l'état de vieillissement du béton armé du quai Lombard.

\begin{tabular}{lcc}
\hline Objet du contrôle & Zones investiguées & Techniques utilisées \\
\hline $\begin{array}{l}\text { Diamètres résiduels des aciers } \\
\text { apparents corrodés }\end{array}$ & Caissons & Mesure directe sur site \\
Carbonatation du béton & Poutres principales et caissons & Mesure directe sur site \\
Potentiels de corrosion des aciers & Poutres principales et caissons & Corrosimètre \\
Profils de concentration en chlorures & Poutres principales & Analyses en laboratoire \\
\hline
\end{tabular}

\subsection{Quai n ${ }^{\circ}$ de Jarry Nord}

Le programme de diagnostic comprenait des éléments destinés à définir l'état des lieux "structure et matériaux" de l’ouvrage, ces éléments ayant été prédéfinis dans le cahier des charges établi par le gestionnaire. Ils comportaient :

- une inspection détaillée complète des parties émergées et immergées avec des mesures d'épaisseur par ultrasons sur les pieux métalliques et la vérification par perçage du niveau des bouchons de béton de remplissage des pieux ;

- la vérification de la géométrie de l'appontement et des flèches des poutres longitudinales par des mesures topométriques ;

- le contrôle dimensionnel des éléments de structure, de la géométrie et des sections des armatures pour valider les données d’entrée ;

- la vérification des caractéristiques de durabilité du béton armé ;

- la vérification par le calcul de la tenue des éléments de structure aux charges d'exploitation, en tenant compte des observations et des mesures faites sur site ;

- la vérification de la tenue aux cyclones et aux séismes, au regard des règles de calcul actuelles.

Tableau 3. Vérification de l'état de vieillissement du béton armé du quai $n^{\circ} 9$ de Jarry

\begin{tabular}{lll}
\hline Objet du contrôle & Zones investiguées & Techniques utilisées \\
\hline \multirow{2}{*}{ Carbonatation du béton } & $\begin{array}{l}\text { Hourdis, âmes de poutres } \\
\text { longitudinales et chevêtres des appuis }\end{array}$ & $\begin{array}{l}\text { Analyses en laboratoire } \\
\text { sur carottes }\end{array}$ \\
Teneur en chlorures et en sulfates & $\begin{array}{l}\text { Hourdis, âmes de poutres } \\
\text { longitudinales et chevêtres des appuis }\end{array}$ & $\begin{array}{l}\text { Analyses en laboratoire } \\
\text { sur cattes }\end{array}$ \\
Densité et porosité du béton & $\begin{array}{l}\text { Hourdis, âmes de poutres } \\
\text { longitudinales et chevêtres des appuis }\end{array}$ & Analyses en laboratoire \\
Examen visuel & Hourdis, âmes de poutres & Stéréomicroscopie sur \\
Dosage de la silice & longitudinales et chevêtres des appuis & carottes \\
& Hourdis, âmes de poutres & Analyses en laboratoire \\
\end{tabular}




\section{XII ${ }^{\text {èmes }}$ Journées Nationales Génie Côtier - Génie Civil \\ Cherbourg, 12-14 juin 2012}

\section{Synthèse des résultats}

\subsection{Extension du quai Modéré Lombard}

Les défauts révélés par l'inspection détaillée sont essentiellement des dégradations des équipements et du matériau béton armé ; ces derniers affectent surtout les caissons préfabriqués du quai et des passerelles; ils se traduisent par des fractures dues à la corrosion des aciers et par des zones d'aciers apparents corrodés parfois jusqu'à rupture. Sur la poutre principale, exposée aux embruns, une décohésion du béton sur environ 1 centimètre d’épaisseur a été observée, sans aciers apparents.
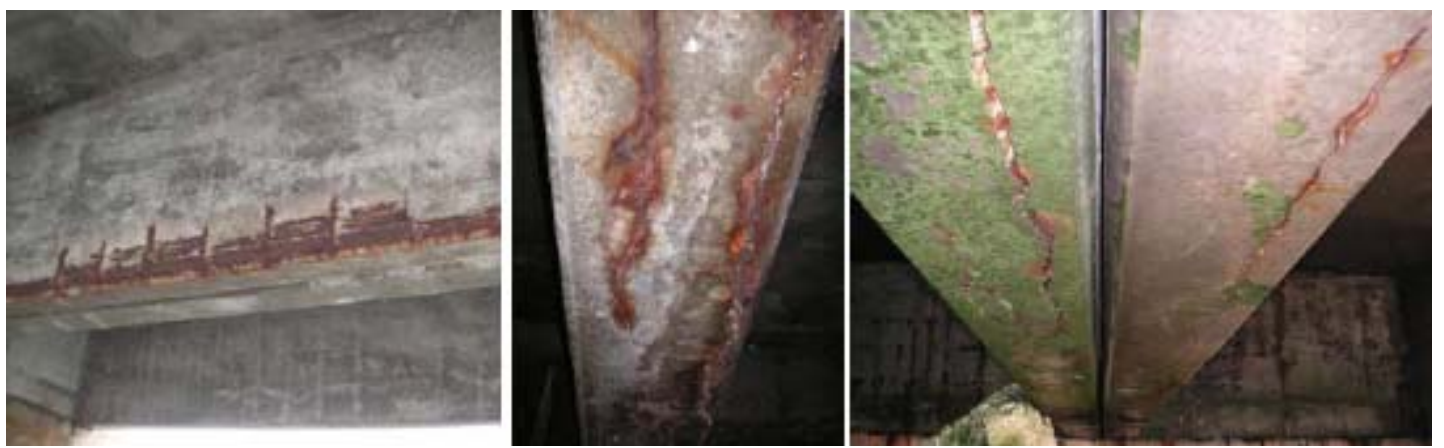

Figure 6. Dégradations du béton armé des caissons du quai Modéré Lombard.

La comparaison entre les données géométriques mesurées sur site et les données des plans d'exécution ne révèle pas d'anomalie, exception faite des épaisseurs d'enrobage qui sont plus faibles que prévues.

L'analyse des mesures faites pour évaluer la durabilité du béton armé des poutres principales montre que, malgré une teneur en chlorures supérieure aux normes en vigueur, le risque de dégradation est relativement faible ; ceci est compatible avec les observations visuelles, qui montrent peu de dégradations sur les poutres principales.

En conclusion, à court terme, sous réserve de la réparation du béton armé des caissons dégradés et, à moins grande échelle, de celui des poutres principales après identification des causes de la décohésion superficielle du béton de la poutre extérieure, la faisabilité du remplacement du portique actuel par un portique de capacité 380 tonnes est avérée. A plus long terme, une réflexion est à mener sur la faisabilité technique et économique de la mise en œuvre d'une protection des poutres vis-à-vis de la corrosion -échloruration, protection cathodique- et sur les actions de suivi préventif nécessaires pour vérifier et garantir le niveau de service de l'extension du quai Modéré Lombard. 


\subsection{Quai $n^{\circ} 9$ de Jarry Nord}

L’inspection détaillée des parties immergées montre que les pieux sont en bon état général, malgré des traces de corrosion visibles de façon plus ou moins étendue. L’inspection détaillée des parties émergées a révélé de nombreux défauts, liés au vieillissement du béton armé (épaufrures, aciers apparents corrodés avec localement rupture des cadres de reprise de l'effort tranchant) mais aussi à des sollicitations importantes de certains éléments de structures (fissuration des poutres et des hourdis).

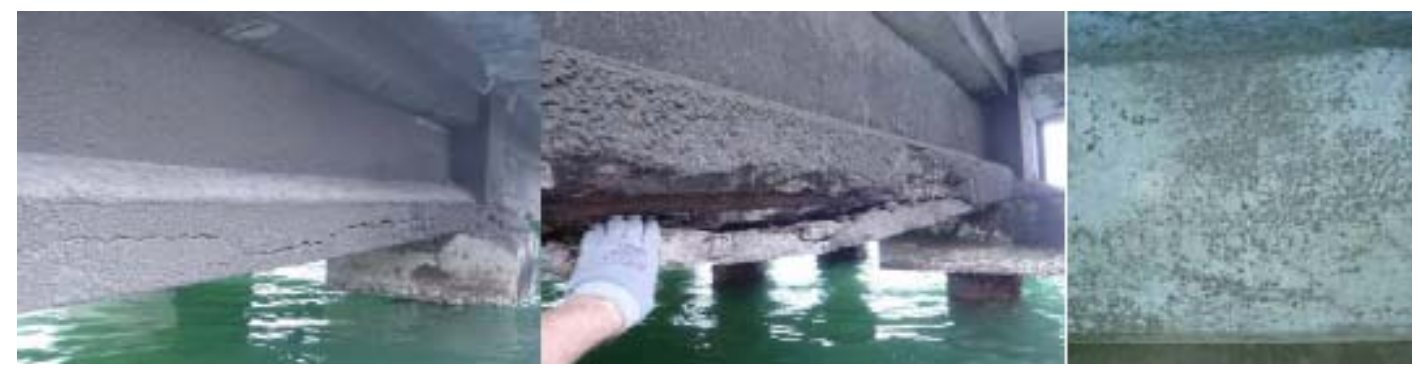

Figure 7. Fracture, aciers corrodés et fissure oblique sur poutres du quai $n^{\circ} 9$ de Jarry.

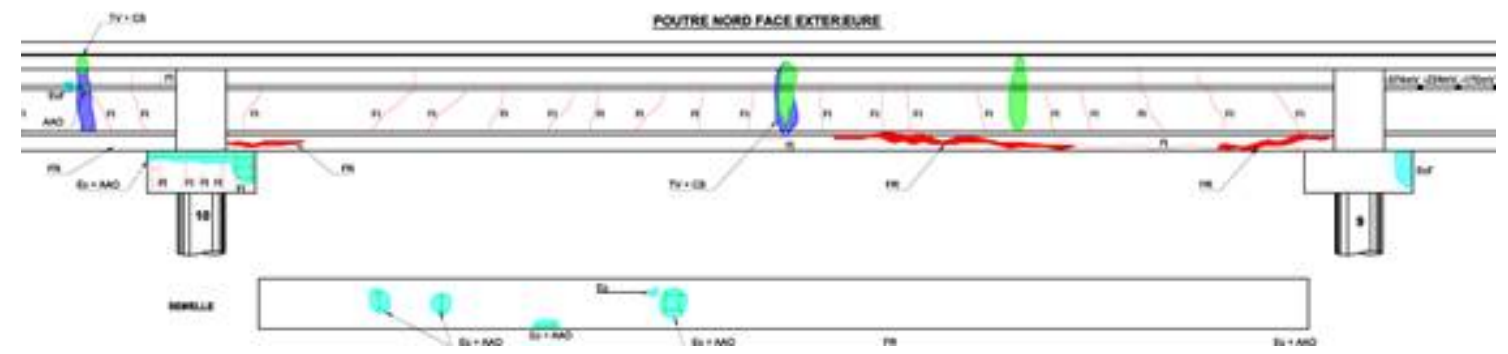

Figure 8. Extrait de la cartographie des dégradations du quai n9 de Jarry.

Le contrôle dimensionnel des éléments de structure n’a pas révélé d'écart avec les plans BPE (bon pour exécution); les sections des aciers mesurées dans les zones où ils sont apparents sont conformes avec ces plans, mais des pertes d'épaisseur de l'ordre de 15 \% dues à la corrosion ont été mesurées.

Les relevés topométriques ont révélés des flèches anormales sur certaines poutres, sans corrélation avec d'autres défauts structuraux.

Les mesures faites sur les prélèvements de béton et sur le site montrent des caractéristiques mécaniques satisfaisantes; en revanche, les teneurs en chlorures sont supérieures à celle de la valeur limite fixée dans la norme NF EN 206-1, et la porosité est relativement importante ; ces deux derniers résultats indiquent une faible résistance à la pénétration des agents agressifs et des risques accrus de corrosion des armatures.

Les calculs, réalisés en tenant compte des données obtenues par les investigations, montrent qu'étant donné la perte de section des armatures par corrosion, le critère de fissuration préjudiciable et le manque de ferraillage de reprise des efforts tranchants, la plupart des poutres longitudinales ne sont pas aptes à subir les charges d'exploitation 


\section{XII ${ }^{\text {èmes }}$ Journées Nationales Génie Côtier - Génie Civil \\ Cherbourg, 12-14 juin 2012}

prévues. Même si, depuis sa mise en service, l'ouvrage a subi sans dommage structurel apparent le passage de Hugo (ouragan de classe 4) et celui d'autres cyclones et tempêtes tropicales de moindre importance, il ne répond ni aux règles actuelles de calcul cyclonique, ni à celles de calcul parasismique, sachant qu'un séisme important -un "big one" similaire à celui survenu en 1843- est attendu.

En conclusion, le quai n ${ }^{\circ} 9$ de Jarry présente des défauts importants tant du point de vue du vieillissement des matériaux que de celui du fonctionnement. Il ne répond ni aux exigences requises pour les charges d'exploitation, ni aux règles parasismiques et cycloniques actuelles. Il est certes toujours en exploitation, mais un certain nombre d'actions sont préconisées pour, dans l'ordre, compléter le diagnostic, remédier aux dégradations, remettre l'ouvrage "aux normes" et lui assurer ainsi une durée de vie optimale.

En ce qui concerne le diagnostic, il est préconisé :

- des analyses matériaux complémentaires pour évaluer la faisabilité technique et économique de la déchloruration qui pourrait améliorer la durabilité du béton armé ;

- des essais de chargement pour s'assurer que les poutres et notamment celles qui présentent des flèches anormales, se comportent correctement vis-à-vis des sollicitations.

La réparation et la mise en conformité de l'ouvrage imposeraient dans tous les cas des travaux lourds, notamment pour satisfaire aux exigences des normes antisismiques actuelles. Le gestionnaire doit donc redéfinir au préalable ses besoins en termes de durée d'exploitation et de niveau de service pour opérer ensuite un choix entre différentes solutions, remplacement de la structure actuelle compris.

Dans la mesure où, dans l'attente de ces actions, l'exploitation de l'ouvrage doit se poursuivre, des limitations d'accès, de tonnage et la mise sous surveillance renforcée par instrumentation ont été préconisées pour prévenir les risques de ruine partielle et de pertes d'exploitation induites.

\section{Conclusion}

Les deux exemples de cas présentés succinctement ici illustrent l'intérêt mais aussi les difficultés des études de diagnostic d'ouvrages anciens menées dans le cadre de projets d'aménagement, d'augmentation de niveau de service ou de prolongation de durée de vie.

Ces études combinent un diagnostic "structure", un diagnostic "matériau" et des calculs de vérification de conformité vis-à-vis des règles d'exploitation actuelles, souvent plus contraignantes que celles en vigueur à l'époque de construction des ouvrages.

Le diagnostic "structure" est établi à partir de l'analyse d'un relevé d'anomalies détectables par inspection visuelle et mesures géométriques locales ou globales; souvent, l'absence de données antérieures aux relevés établis lors du diagnostic, empêche l'évaluation du caractère évolutif de certaines anomalies, déterminante pour 
appréhender leur cause et leur gravité. Le diagnostic "matériau" -dont le référentiel technique et normatif n'est pas encore entièrement stabilisé- fait appel à des techniques de contrôle non destructif et à des analyses sur prélèvements dont la mise en œuvre et l'interprétation sont souvent délicates ; le choix des zones à investiguer, des paramètres physico-chimiques pertinents et d'un échantillonnage représentatif ne peut être efficacement opéré qu'après une analyse préliminaire qui comprend a minima une visite du site et l'examen des données d'entrée. Les calculs de vérification de conformité intègrent les données recueillies par le diagnostic "structure" et "matériau" avec des hypothèses et des simplifications souvent réductrices par rapport à l'état réel des structures.

Il peut donc subsister, à l'issue d'une étude de diagnostic, des inconnues sur l'étendue des dégradations, leur origine ou leur gravité. La levée de ces inconnues, nécessaire à la définition de préconisations dont la faisabilité technique et économique doit être évaluée au regard des exigences et des contraintes du gestionnaire, nécessite la mise en œuvre d'investigations complémentaires. Ces investigations comprennent souvent des analyses sur les matériaux -par exemple, des mesures étendues de profils de concentration en ions chlorures dans le béton pour évaluer la pertinence d'une déchloruration ou d'une protection cathodique- et des essais de chargement, afin de vérifier le comportement réel de la structure ou d'éléments de structure sous sollicitations contrôlées.

Si le diagnostic donne des éléments objectifs de réponse sur les conséquences possibles de non-conformités avérées -ruine partielle ou totale, apparition de graves désordres, réduction du niveau de service, pertes d'exploitation-, l'ingénierie forensique et les méthodes de gestion par les risques peuvent apporter une aide complémentaire à la décision pour le gestionnaire, qui doit parfois faire face à des choix techniques, stratégiques et budgétaires difficiles. Ces méthodes, appliquées principalement dans l'industrie, connaissent depuis peu des applications dans le domaine de la maintenance des structures de génie civil ; elles permettent de quantifier les liens et les interactions entre les actions de maintenance et les risques techniques, économiques, environnementaux et sécuritaires associés à l'exploitation des structures.

Dans tous les cas, l'existence d'un suivi organisé intégrant l'évaluation régulière des paramètres qui caractérisent l'état et le fonctionnement des structures permet de définir au mieux le programme de diagnostic, de limiter les inconnues résiduelles et d'établir des préconisations qui faciliteront le choix puis la mise au point de solutions adaptées à la problématique posée. 